\title{
Temperature dependence of large exchange-bias in TbFe-Co/Pt
}

\author{
S. Romer, ${ }^{1}$ M. A. Marioni, ${ }^{1, a)}$ K. Thorwarth, N. R. Joshi, ${ }^{1}$ C. E. Corticelli, ${ }^{1}$ H. J. Hug, ${ }^{1, b)}$ \\ S. Oezer, ${ }^{2}$ M. Parlinska-Wojtan, ${ }^{3}$ and H. Rohrmann ${ }^{4}$ \\ ${ }^{1}$ Empa, Swiss Federal Institute for Materials Testing and Research, CH-8600 Dübendorf, Switzerland \\ ${ }^{2}$ Department of Physics, Universität Basel, CH-4056 Basel, Switzerland \\ ${ }^{3}$ Institute of Nanotechnology, University of Rzeszow, 35-959 Rzeszow, Poland \\ ${ }^{4}$ Oerlikon Balzers, 9496 Balzers, Liechtenstein.
}

(Received 27 July 2012; accepted 29 October 2012; published online 27 November 2012)

\begin{abstract}
The exchange-bias effect provides unidirectional anisotropy to ferromagnetic thin-films in GMR and TMR sensors. It is weak because it relies on an inherently compensated coupling across a ferromagnet/antiferromagnet interface. We replace the antiferromagnet with a TbFe-based perpendicular-anisotropy amorphous ferrimagnet, coupling to an adjacent ferromagnet with $13-5 \mathrm{~mJ} / \mathrm{m}^{2}$ for temperatures within $7.9-280 \mathrm{~K}$. A large exchange-bias between 1.1 and $0.7 \mathrm{~T}$ in that temperature range ensues. The temperature dependence of the bias is step-like, and thus different from that of antiferromagnet-based exchange-bias systems. It suggests using this material in exchange-biased temperature assisted magnetic writing. () 2012 American Institute of Physics. [http://dx.doi.org/10.1063/1.4767142]
\end{abstract}

Giant magnetoresistance (GMR) and tunnel magnetoresistance (TMR) devices ${ }^{1,2}$ require a thin film with a fixed magnetization direction to operate. It is provided by a ferromagnetic film whose magnetization has been exchangebiased $^{3}$ in one direction by an adjacent antiferromagnetic film. Biasing arises from anchoring the ferromagnet's magnetization in the antiferromagnet, by coupling it to uncompensated spins ${ }^{4}$ of the latter, which remain pinned in applied fields ${ }^{5}$ and sufficiently low temperatures. In practice, the coupling strength and consequently also the exchange-bias are weak.

One way to increase the exchange-bias is to raise the density of pinned uncompensated amplitude freedom (AF) spins, ${ }^{6}$ e.g., by placing defects in the antiferromagnet. ${ }^{7,8}$ Alternatively, non-antiferromagnetic, high-coercivity materials ${ }^{9-14}$ could also anchor ferromagnetic magnetization in place of antiferromagnets, provided the inter-layer coupling is sufficiently strong. Thus, $0.46 \mathrm{~T}$ exchange-bias has been observed in ferrimagnetic-ferromagnetic exchange spring systems ${ }^{9}$ with in-plane magnetization.

In this work, we report the observation of large exchange-bias in a perpendicular system using an amorphous $\mathrm{Tb}-\mathrm{Fe}$ ferrimagnet and a $\mathrm{Co} / \mathrm{Pt}$ multilayer. Because the $\mathrm{Tb}$ moments are expected to align antiparallel to the $\mathrm{Fe}$ and $\mathrm{Co}$ moments, and, consistently, the Fe moments would align parallel to Co ones, we expect the ferrimagnetic-ferromagnetic coupling to be largely frustration-free and hence strong. We fabricated amorphous- $\mathrm{Tb}_{23} \mathrm{Fe}_{77}$-based thin-film structures by DC magnetron sputtering at room temperature from $\mathrm{Tb}_{20} \mathrm{Fe}_{80}$ micro-mosaic targets (Mitsubishi Materials Corp., Tokyo, Japan) using Ar gas at $5 \times 10^{-3}$ mbar. A first sample (sample 1) consists of a $\mathrm{Tb}_{23} \mathrm{Fe}_{77}$-ferrimagnetic layer (specifically, $\mathrm{Si}^{\text {nat }} \mathrm{Pt}_{5 \mathrm{~nm}} \mathrm{TbFe}_{40 \mathrm{~nm}} \mathrm{Pt}_{10 \mathrm{~nm}}$ ). A second sample (sample 2) comprises a ferrimagnet-ferromagnet bilayer $\left(\mathrm{Si}^{\text {nat }} \mathrm{Pt}_{5 \mathrm{~nm}} \mathrm{TbFe}_{40 \mathrm{~nm}}\right.$ $\left.\left[\mathrm{Co}_{0.4 \mathrm{~nm}} \mathrm{Pt}_{0.7 \mathrm{~nm}}\right]_{\times 5} \mathrm{Pt}_{10 \mathrm{~nm}}\right)$. For reference, we also sputtered a

\footnotetext{
a)Miguel.Marioni@empa.ch.

b)Also at Department of Physics, Universität Basel, CH-4056 Basel, Switzerland.
}

ferromagnetic multilayer $\left(\mathrm{Si}^{\text {nat }} \mathrm{Pt}_{5 \mathrm{~nm}}\left[\mathrm{Co}_{0.4 \mathrm{~nm}} \mathrm{Pt}_{0.7 \mathrm{~nm}}\right]_{\times 5} \mathrm{Pt}_{10 \mathrm{~nm}}\right.$, sample 3) and a conventional exchange-bias system $\left(\mathrm{Si}^{\text {nat }} \mathrm{Pt}_{5 \mathrm{~nm}} \mathrm{MnIr}_{3.4 \mathrm{~nm}}\left[\mathrm{Co}_{0.4 \mathrm{~nm}} \mathrm{Pt}_{0.7 \mathrm{~nm}}\right]_{\times 5} \mathrm{Pt}_{2 \mathrm{~nm}}\right.$, sample 4 , where MnIr was sputtered from a target with nominal composition $\mathrm{Mn}_{75} \mathrm{Ir}_{25}$ ). All samples were deposited onto Si (100) wafers with a native oxide layer, denoted $\mathrm{Si}^{\text {nat }}$.

Figure 1(a) shows perpendicular magnetization hysteresis loops obtained by vibrating sample magnetometry at selected temperatures for sample 1, i.e., the ferrimagnet only. The measurements were carried out after heating the film to $400 \mathrm{~K}$ and subsequently cooling down in a $+7 \mathrm{~T}$ field. At the desired temperature, the field was lowered from $+7 \mathrm{~T}$ to $-7 \mathrm{~T}$ and then raised back to $+7 \mathrm{~T}$.

The loops have the square shape common for perpendicular anisotropy materials. A departure from that type of behavior is most visible in the $280 \mathrm{~K}$ loop. It indicates that a fraction of the film has an in-plane anisotropy and remains coupled to the part of the film with perpendicular magnetization. Figure 1(b) summarizes the main magnetic characteristics of sample 1 up to $280 \mathrm{~K}$. This temperature range is of particular interest because in it, the coercivity exceeds $3 \mathrm{~T}$ : From a minimum of $3.1 \mathrm{~T}$ at $215 \mathrm{~K}$, it increases toward lower temperatures, as well as toward the compensation temperature $T_{\text {comp }} \approx 320 \mathrm{~K}$. The remanence decreases toward zero approaching $T_{\text {comp }}$. One can estimate a lower bound for the magnetic anisotropy in terms of the coercivity $H_{c}$ and the magnetic moment per unit area $m / A$ in the material as $K_{u}^{\text {est. }}=2 M_{R} H_{c}$, with $M_{R}=(m / A) / d$ (here, $d$ is the magnetic film thickness). Accordingly, the anisotropy takes on values in the green shaded region of Fig. 1(b), which are consistent with previous observations. ${ }^{15}$

Sample 1's large coercivity and anisotropy suggest the TbFe-ferrimagnet could pin the magnetization of ferromagnetic layers coupled to it. The hypothesis can be tested using sample 2, by maintaining its ferrimagnetic layer magnetized in one direction and studying the switching of the ferromagnetic film adjacent to it. As with sample 1, we heat sample 2 to $400 \mathrm{~K}$, subsequently cooling it in a $+7 \mathrm{~T}$ field. This field 
(a)

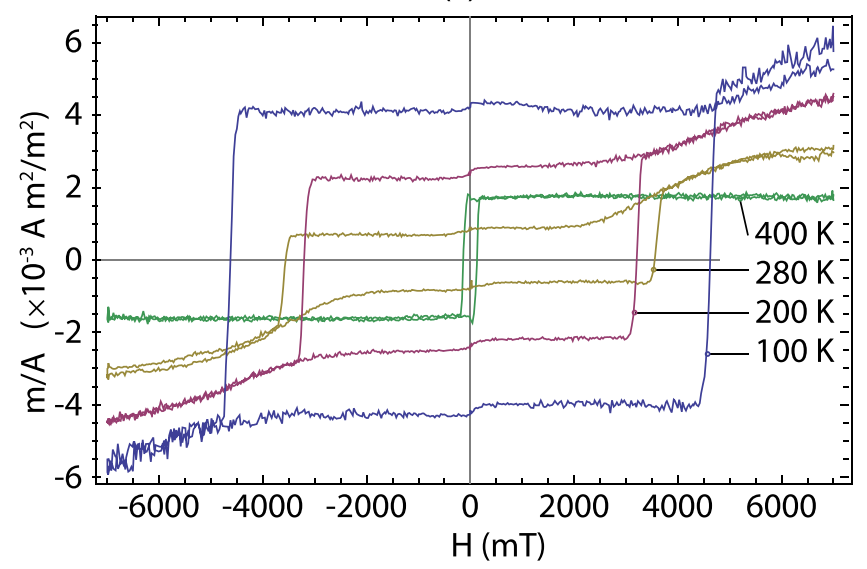

(b)

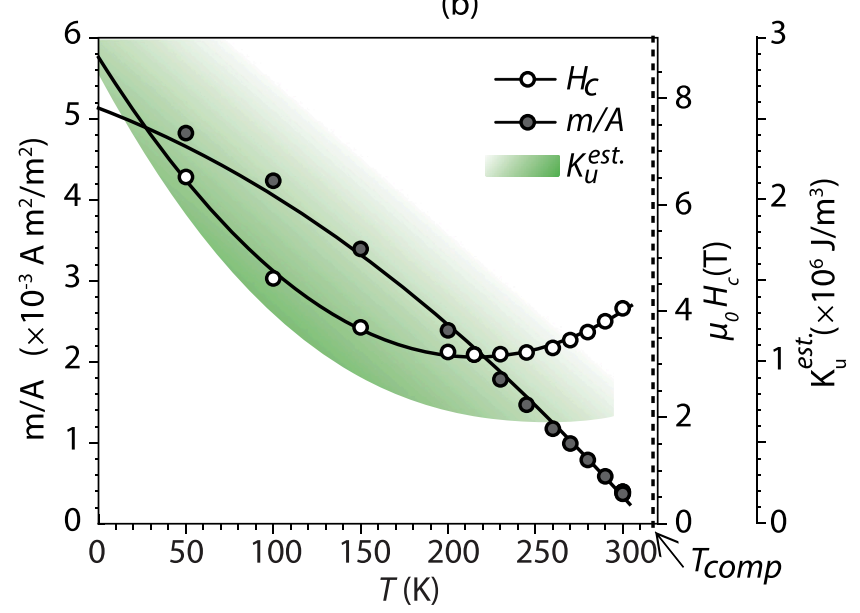

FIG. 1. Magnetometry of $\mathrm{Pt}_{5 \mathrm{~nm}}\left(\mathrm{~Tb}_{23} \mathrm{Fe}_{77}\right)_{40 \mathrm{~nm}} \mathrm{Pt}_{10 \mathrm{~nm}}$, sample 1. (a) Hysteresis loops at selected temperatures. A predominantly square loop shape is consistent with the overly perpendicular anisotropy of the films. (b) Summary of the hysteresis loop characteristics between $50 \mathrm{~K}$ and $300 \mathrm{~K}$. Filled circles: magnetic moment areal density, $m / A$; Empty circles: coercivity; The solid lines are a guide for the eye. Shaded area: implied lower bound for the magnetic anisotropy. Solid lines are a guide for the eye.

exceeds the coercivity of either sublayer, and in particular that of the ferrimagnet (at least during passage through temperatures above $\approx 50 \mathrm{~K}-\mathrm{cf}$. Fig. 1(b)). Therefore, with the possible exception of the interface region, the ferrimagnet magnetization (and also that of the ferromagnetic $\mathrm{Co} / \mathrm{Pt}$ multilayer) will be aligned parallel to the applied field, i.e., in the + direction. Cycling the field at the desired temperature from $+7 \mathrm{~T}$ to a large negative field and back could reverse the ferrimagnet's "+" magnetization. To avoid that, we select a largest negative excursion of the magnetic field that is lower than the ferrimagnet coercivity (cf. Fig. 1(b)). Except for the lowest studied temperature, $7.9 \mathrm{~K}$ (not shown), at which the ferrimagnet coercivity could be estimated to exceed the maximum field attainable with our magnetometer, we chose $-2 \mathrm{~T}$ as the largest negative field excursion.

Figure 2 shows magnetization loops thus obtained for the same temperatures reported upon in Fig. 1. At $400 \mathrm{~K}$, the hysteresis loop has a rectangular shape typical for perpendicular anisotropy magnetization loops, but at the other temperatures, the loop is asymmetric. The magnetization presents a "step" at $H_{\text {step }}$, in the vicinity of $+1 \mathrm{~T}$, which is absent at $-H_{\text {step }}$.

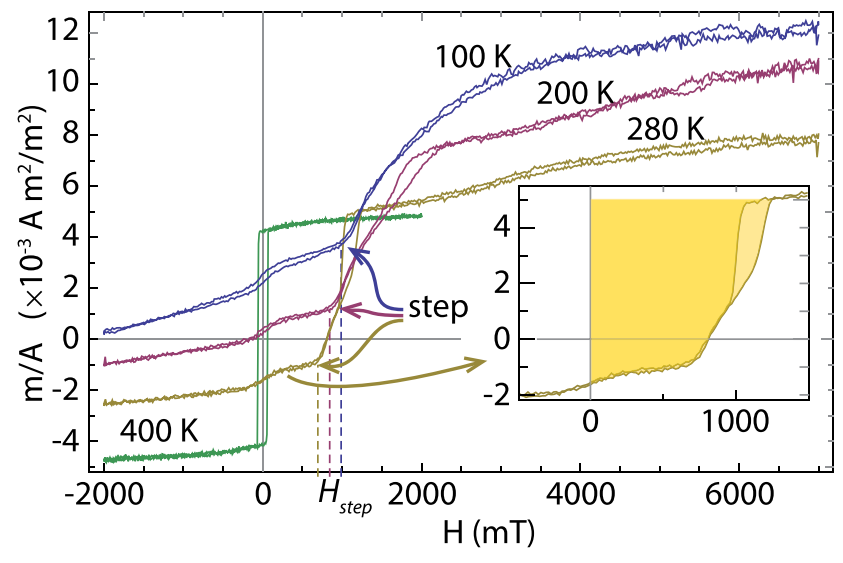

FIG. 2. Magnetometry of $\mathrm{TbFe}_{40 \mathrm{~nm}}\left[\mathrm{Co}_{0.4 \mathrm{~nm}} \mathrm{Pt}_{0.7 \mathrm{~nm}}\right]_{\times 5}$, sample 2, at selected temperatures. Inset: zoom-in on the $280 \mathrm{~K}$ loop. The shaded area measures the energy required to magnetize the ferromagnet at that temperature, and estimates the strength of its coupling to the ferrimagnet.

Further, the loops are staggered vertically: $m / A(-2 T)$ is positive for the lowest temperatures and decreases to negative values as the temperature increases.

Note that $H_{\text {step }}$ is significantly smaller than the ferrimagnet's coercivity, and also the amplitude of the step in the magnetization loops below $400 \mathrm{~K}$ is comparable to twice the magnetic moment areal density in a saturated $\left[\mathrm{Co}_{0.4 \mathrm{~nm}}\right.$ $\left.\mathrm{Pt}_{0.7 \mathrm{~nm}}\right]_{\times 5}$ film (not shown). We can, therefore, identify the step at $H_{\text {step }}$ with switching of the Co/Pt multilayer. The shift to positive fields of the $\mathrm{Co} / \mathrm{Pt}$ switching is due to its coupling to the "pinned" ferrimagnet, and thus comparable to exchange-bias in ordinary antiferromagnet-based exchangebias systems. It represents the largest bias found to date (a thinner ferromagnet would lead to larger exchange-bias, e.g., $2 \mathrm{~T}$ for $\mathrm{Si}^{\text {nat }} \mathrm{Pt}_{5 \mathrm{~nm}} \mathrm{TbFe}_{40 \mathrm{~nm}}\left[\mathrm{Co}_{0.4 \mathrm{~nm}} \mathrm{Pt}_{0.7 \mathrm{~nm}}\right]_{\times 2} \mathrm{Pt}_{10 \mathrm{~nm}}$, not shown). The $\mathrm{Co} / \mathrm{Pt}$ multilayer is magnetized in the - direction at $H<H_{\text {step }}$ and + at $H>H_{\text {step }}$. At temperatures below the compensation, i.e., below $\approx 320 \mathrm{~K}$, the $\mathrm{Tb}$ moments determine the ferrimagnet's net magnetization direction, and, therefore, the $\mathrm{Tb}$ moments are pointed in the + direction throughout the measurements, except for the $400 \mathrm{~K}$ loop. Co spins coupled to the $\mathrm{Tb}$ spins will, therefore, align antiparallel to the latter (like the Fe spins in the ferrimagnet) until they are switched by an applied field. Therefore, in negative fields, the net magnetic moment will be the sum of the positive $\mathrm{TbFe}-\mathrm{moment}$ and the negative $\mathrm{Co} / \mathrm{Pt}$ moment. Below about $200 \mathrm{~K}$, the former dominates the sum, and the measured magnetic moment is positive. Conversely, at higher temperatures, the moment is dominated by $\mathrm{Co} / \mathrm{Pt}$, resulting in a negative net magnetic moment. This behavior is apparent in the vertically shifted loops of Fig. 2.

The fact that $\mathrm{Co} / \mathrm{Pt}$ switches into alignment with the field at comparatively large values of $H_{\text {step }}$ implies a strong interlayer coupling. We can estimate a lower bound for the coupling energy from the magnetization loop, e.g., for $280 \mathrm{~K}$, as the area shaded in the inset of Fig. 2. Thus, the coupling energy lower bound amounts to $5 \pm 1 \mathrm{~mJ} / \mathrm{m}^{2}$ (the $\pm 1 \mathrm{~mJ} / \mathrm{m}^{2}$ departure reflects the loop hysteresis) at $280 \mathrm{~K}$, increasing to $13 \pm 1 \mathrm{~mJ} / \mathrm{m}^{2}$ at $7.9 \mathrm{~K}$. These levels are comparable to values found in exchange-coupled rare earth-ferrimagnet double layers ${ }^{11}$ and exceed twice the values of $\mathrm{Mn}_{3}$ Ir-based systems with improved ordering. ${ }^{16}$ 


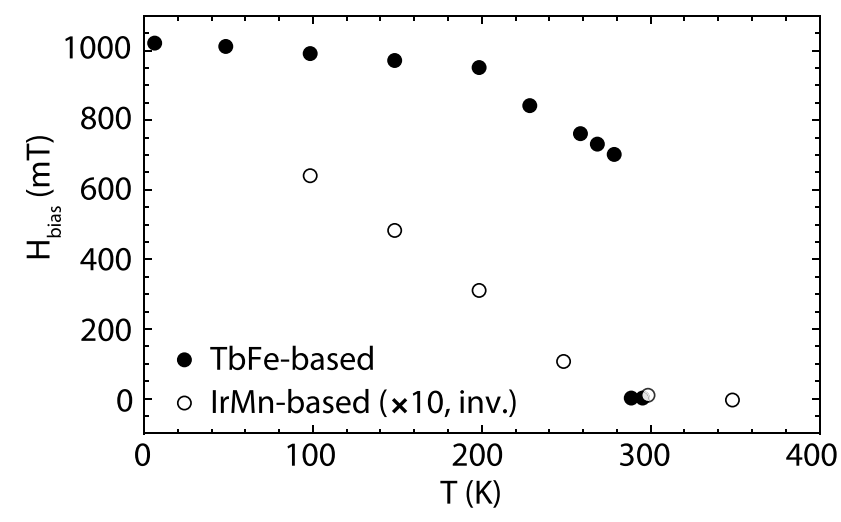

FIG. 3. Exchange-bias as a function of temperature for a conventional MnIr-based system, sample 3 (empty markers) and a TbFe-ferrimagnet based system, sample 2 (black markers). For the ease of comparison, the MnIr-system data have been inverted and expanded by a factor of 10 .

Figure 3 presents a summary of the exchange-bias observation in sample 2 in the temperature range $7.9-280 \mathrm{~K}$, described by $H_{\text {step }}$ (black markers), as given by the measurements of Fig. 2 and similar ones at intermediate temperatures (not shown). Upon cooling exchange-bias appears at $280 \mathrm{~K}$, with a value of $H_{\text {step }}=0.7 \mathrm{~T}$. A monotonic increase of $H_{\text {step }}$ is seen as the temperature is lowered in the range $7.9-280 \mathrm{~K}$. $H_{\text {step }}$ appears to saturate near $1.1 \mathrm{~T}$ at $T \approx 7.9 \mathrm{~K}$. In contrast, $H_{e x}$ in conventional exchange-bias systems shows no such saturation. $H_{e x}$ in those systems increases continually as the temperature decreases, with constant or increasing rate. Such systems typically show the evolution of exchange-bias with temperature seen for sample 4 (empty markers), which employs antiferromagnetic MnIr anchor for the ferromagnet's magnetization. For ease of comparison, we show inverted values of $H_{e x}$ and use a magnified scale: $10 \times H_{e x}$.

Note that the magnitude of the exchange-bias in TbFe-based systems is an order of magnitude larger than in conventional systems. This observation is consistent with the view that there is no frustration of the coupling of the $\mathrm{TbFe}$ and the $\mathrm{Co} / \mathrm{Pt}$ layers, whereas it can be significant in the MnIr-based case (in particular, the number of pinned uncompensated antiferromagnet spins is small ${ }^{17}$ ). Further, the comparatively weak temperature dependence of bias in sample 2 is consistent with "pinning" in the ferrimagnet arising from its anisotropy. This is different from the antiferromagnetbased sample 4, where we would expect thermally activated switching of antiferromagnetic domains to strongly influence the degree of pinning of the uncompensated antiferromagnetic spins. $^{18,19}$

Thus, we have shown a perpendicular magnetization system based on an amorphous $\mathrm{TbFe}$ ferrimagnet and a $\mathrm{Co} / \mathrm{Pt}$ multilayer ferromaget with large inter-layer coupling and exchange-bias of the order of $1 \mathrm{~T}$. The observation of the temperature dependence of the exchange-bias supports the notion that bias is limited by the coercivity of the ferrimagnet. It is foreseeable that employing these ferrimagnet-based exchangebias systems should lead to more sensitive and robust magnetic sensors. ${ }^{20}$ Furthermore, appropriate engineering of the compensation temperature for the ferrimagnet (cf. Ref. 21) could lead to useful magnetic recording materials. Specifically, they might circumvent the difficulties of writing weakly magnetized materials in thermally assisted recording ${ }^{22}$ systems by placing the competing high-temperature requirements of low anisotropy and large magnetization ${ }^{23}$ on different layers of a ferrimagnet-ferromagnet medium. In such a storage technique, information would be stored at room temperature in the ferrimagnet, which, on account of its high coercivity and low magnetic moment would warrant the thermal stability of the information. A soft ferromagnet strongly coupled to it would enhance the stray field for readout. By retaining sufficient magnetization at higher temperatures, where the ferrimagnet has low coercivity, the ferromagnet would also provide the lever to write the ferrimagnet using the strong coupling.

We would like to thank M. Döbeli for carrying out RBS measurements to determine the composition of our $\mathrm{TbFe}$ samples, J. Patscheider, S. Roos, and J. Seif for help with the deposition system setup. This work was supported by Empa, the NCCR Nanoscale Science of the University of Basel, and SNF Grant 200021-117970. In Memoriam Dr. Lorenzo Castaldi 17.6.1972 - 25.3.2012.

${ }^{1}$ M. N. Baibich, J. M. Broto, A. Fert, F. Nguyen Van Dau, F. Petroff, P. Etienne, G. Creuzet, A. Friederich, and J. Chazelas, Phys. Rev. Lett. 61, 2472 (1988).

${ }^{2}$ G. Binasch, P. Grünberg, F. Saurenbach, and W. Zinn, Phys. Rev. B 39, 4828 (1989).

${ }^{3}$ W. H. Meiklejohn and C. P. Bean, Phys. Rev. 102, 1413 (1956).

${ }^{4}$ K. Takano, R. H. Kodama, A. E. Berkowitz, W. Cao, and G. Thomas, Phys. Rev. Lett. 79, 1130 (1997).

${ }^{5}$ P. Kappenberger, S. Martin, Y. Pellmont, H. J. Hug, J. B. Kortright, O. Hellwig, and E. E. Fullerton, Phys. Rev. Lett. 91, 267202 (2003).

${ }^{6}$ I. Schmid, M. A. Marioni, P. Kappenberger, S. Romer, M. Parlinska-Wojtan, H. J. Hug, O. Hellwig, M. J. Carey, and E. E. Fullerton, Phys. Rev. Lett. 105, 197201 (2010).

${ }^{7}$ P. Miltényi, M. Gierlings, J. Keller, B. Beschoten, G. Güntherodt, U. Nowak, and K. D. Usadel, Phys. Rev. Lett. 84, 4224 (2000).

${ }^{8}$ D. Martien, K. Takano, A. E. Berkowitz, and D. Smith, Appl. Phys. Lett. 74, 1314 (1999).

${ }^{9}$ E. E. Fullerton, J. S. Jiang, M. Grimsditch, C. H. Sowers, and S. D. Bader, Phys. Rev. B 58, 12193 (1998).

${ }^{10}$ S. Mangin, G. Marchal, and B. Barbara, Phys. Rev. Lett. 82, 4336 (1999).

${ }^{11}$ C.-C. Lin, C.-H. Lai, R.-F. Jiang, and H.-P. D. Shieh, J. Appl. Phys. 93, 6832 (2003).

${ }^{12}$ X. Chen, K. Q. Wang, P. Hor, Y. Xue, and C. Chu, Phys. Rev. B 72, 054436 (2005).

${ }^{13}$ K. Dumesnil, C. Dufour, S. Fernandez, M. Oudich, A. Avisou, A. Rogalev, and F. Wilhelm, J. Phys.: Condens. Matter 21, 236002 (2009).

${ }^{14} \mathrm{M}$. Ungureanu, K. Dumesnil, C. Dufour, N. Gonzalez, F. Wilhelm, A. Smekhova, and A. Rogalev, Phys. Rev. B 82, 174421 (2010).

${ }^{15}$ R. B. van Dover, M. Hong, E. M. Gyorgy, J. F. Dillon, and S. D. Albiston, J. Appl. Phys. 57, 3897 (1985).

${ }^{16}$ M. Tsunoda, K. Imakita, M. Naka, and M. Takahashi, J. Magn. Magn. Mater. 304, 55 (2006).

${ }^{17}$ E. Blackburn, C. Sanchez-Hanke, S. Roy, D. J. Smith, J.-I. Hong, K. T. Chan, A. E. Berkowitz, and S. K. Sinha, Phys. Rev. B 78, 180408 (2008).

${ }^{18}$ E. Fulcomer and S. Charap, J. Appl. Phys. 43, 4190 (1972).

${ }^{19}$ K. O'Grady, L. Fernandez-Outon, and G. Vallejo-Fernandez, J. Magn. Magn. Mater. 322, 883 (2010).

${ }^{20}$ F. Radu, R. Abrudan, I. Radu, D. Schmitz, and H. Zabel, Nat. Commun. 3, 715 (2012).

${ }^{21}$ Y. Mimura, N. Imamura, T. Kobayashi, A. Okada, and Y. Kushiro, J. Appl. Phys. 49, 1208 (1978).

${ }^{22}$ R. Rottmayer, S. Batra, D. Buechel, W. Challener, J. Hohlfeld, Y. Kubota, L. Li, B. Lu, C. Mihalcea, K. Mountfield, K. Pelhos, C. Peng, T. Rausch, M. Seigler, D. Weller, and X. Yang, IEEE Trans. Magn. 42, 2417 (2006).

${ }^{23}$ R. F. L. Evans, R. W. Chantrell, U. Nowak, A. Lyberatos, and H.-J. Richter, Appl. Phys. Lett. 100, 102402 (2012). 